\title{
Adaptación peruana del Minimental State Examination
} (MMSE)

\author{
Peruvian adaptation of the Minimental State Examination (MMSE)
}

\author{
Nilton Custodio ${ }^{1,2,3}$, David Lira ${ }^{1,2,3}$ \\ Unidad de Diagnóstico de Deterioro Cognitivo y Prevención de Demencia. Clínica Internacional, Lima, Perú. \\ ${ }^{2}$ Unidad de Investigación, Instituto Peruano de Neurociencias, Lima, Perú. \\ ${ }^{3}$ Unidad de Investigación. Clínica Internacional, Lima, Perú.
}

\section{SEÑOR EDITOR:}

El Minimental State Examination (MMSE) es una de las pruebas cognitivas breves más utilizadas en el mundo para el tamizaje de pacientes con quejas cognitivas. El MMSE fue diseñado originalmente en inglés ${ }^{(1)}$, y posteriormente validado al español.

Nuestro grupo de investigación ha realizado numerosas publicaciones y trabajos de investigación empleando la adaptación peruana del MMSE, en base a la versión 'rioplatense' del MMSE de Butman y col. (2); sin embargo, no hemos publicado dichas modificaciones.

Debido a múltiples consultas recibidas de investigadores peruanos acerca de las adaptaciones realizadas al MMSE versión de Butman, que fue empleada en la realización del estudio de prevalencia de demencia en una población urbana de Lima publicado el año 2008 en la Revista Anales de la Facultad de Medicina ${ }^{(3)}$; procedemos a enunciar las adaptaciones realizadas:

1. En el ítem repetición se cambió la frase: "el flan tiene frutillas y frambuesas" por la frase: "la mazamorra morada tiene duraznos y guindones".
2. En el ítem orden de tres comandos se cambió la orden: "Tome este papel con la mano izquierda, dóblelo por la mitad y póngalo en el suelo" por la orden: "Tome este papel con la mano izquierda, dóblelo por la mitad y devuélvamelo con la mano derecha".

Desde el año 2008, la adaptación peruana del MMSE ha sido empleada por nuestro grupo de investigación en numerosos estudios ${ }^{(4-6)}$, sin dificultad alguna.

Para los investigadores interesados en obtener la adaptación peruana del MMSE, la misma se encuentra disponible a solicitud al correo electrónico del responsable de la correspondencia.

\section{REFERENCIAS BIBLIOGRÁFICAS}

1. Folstein M, Folstein S, McHugh P. "Mini-Menta State" a practical method for grading the cognitive state of patients for the clinician. J Psych Res. 1975;12;189-98.

2. Butman J, Arizaga R, Harris P, Drake M, Baumann Denise, de Pascale A, Allegri R, et al. El "Mini - Mental State Examination" en español. Normas para Buenos Aires. Rev Neurol Arg. 2001;26:11-5.
3. Custodio N, García A, Montesinos R, Escobar J, Bendezú L. Prevalencia de demencia en una población urbana de Lima-Perú: estudio puerta a puerta. An Fac med. 2008;69,233-8.

4. Custodio N, García A, Montesinos R, Lira D, Bendezú L. Validación de la prueba de dibujo del reloj - versión de Manos - como prueba de cribado para detectar demencia en una población adulta mayor de Lima, Perú. Rev Peru Med Exp Salud Publica. 2011;28:29-34.

5. Custodio N, Lira D, Montesinos R, Gleichgerrcht E, Manes F. Utilidad del Addenbrooke's Cognitive Examination versión en español en pacientes peruanos con enfermedad de Alzheimer y demencia frontotemporal. Vertex. 2012;23:165-72.

6. Herrera-Pérez E, Custodio N, Lira D, Montesinos R, Bendezu L. Validity of Addenbrooke's cognitive examination to discriminate between incipient dementia and depression in elderly patients of a private clinic in Lima, Peru. Dement Geriatr Cogn Disord Extra. 2013;3:333-41.

Fuente de financiamiento:

Ninguno

Conflictos de interés:

Ninguno

Autor corresponsal:

Nilton Custodio.

Servicio de Neurologia, Instituto Peruano de Neurociencias, IPN

Jr. Bartolomé Herrera 161, Lince, Lima 14, Perú

Telf: (511) 2653834

Correo electrónico: ncustodio@ipn.pe 